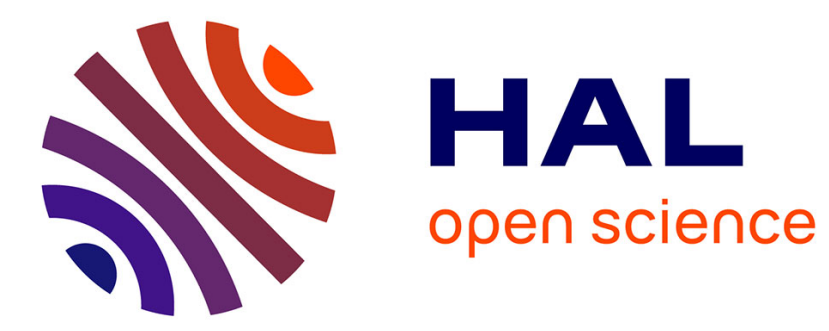

\title{
Suggestion pour un parc national
}

Louis Duplaquet

\section{To cite this version:}

Louis Duplaquet. Suggestion pour un parc national. Revue forestière française, 1954, 8-9, pp.549-550.

10.4267/2042/27019 . hal-03384195

\section{HAL Id: hal-03384195 \\ https://hal.science/hal-03384195}

Submitted on 18 Oct 2021

HAL is a multi-disciplinary open access archive for the deposit and dissemination of scientific research documents, whether they are published or not. The documents may come from teaching and research institutions in France or abroad, or from public or private research centers.
L'archive ouverte pluridisciplinaire HAL, est destinée au dépôt et à la diffusion de documents scientifiques de niveau recherche, publiés ou non, émanant des établissements d'enseignement et de recherche français ou étrangers, des laboratoires publics ou privés. 


\section{SUGGESTION POUR UN PARC NATIONAL}

La protection de la nature est à l'ordre du jour. On connait la propagande menée à son sujet par M. le Professeur Heim, du Muséum d'Histoire Naturelle, par M. le Directeur Ph. Guinier, par l'Union Internationale pour la Protection de la Nature. La Revue Forestière Française y a consacré un fascicule entier $\mathrm{n}^{\circ}$ I 2 de décembre I950).

De tout ceci il ressort que la France métropolitaine est encore bien pauvre en réserves naturelles intégrales et en parcs nationaux. La cause essentielle vient de ce qu'il s'agit d'un pays de vieille civilisation, à peu près complètement occupé et, dans l'ensemble, convenablement mis en valeur. Il n'en reste pas moins que certaines régions incultivables et pittoresques devraient pouvoir entrer dans le cadre défini par la Conférence internationale de Londres de novembre I933. Les fameuses Gorges du Tarn, entre Sainte-Enimie et Le Rosier, sembleraient tout indiquées pour constituer un véritable parc national. Elles rappellent, sur un modèle plus réduit et moins impressionnant, le Grand Canon du Colorado (Etat d'Arizona) que le rédacteur de ces lignes a eu le privilège de pouvoir contempler en I93I.

La vallée du Tarn, dans la partie considérée, ne doit guère être capahle de nourrir ses habitants, sauf ceux qui s'adonnent au commerce de l'hôtellerie: mais ce genre d'activité est parfaitement admis dans les parcs nationaux, tout au moins jusqu'à de certaines limites. Les cultures sont inexistantes, si on excepte les quelques malheureux pieds de vigne qui s'accrochent désespéréement au rocher et dont le rendement économique apparaît plus que dérisoire, à une époque surtout où le marché du vin s'effondre. Aussi la population locale fuit-elle sous d'autres cieux à la recherche d'un travail plus rémunérateur; des maisons abandonnées s'écroulent. Cet exode ne fera sans doute que s'amplifier avec les exigences de la vie moderne. C'est le moment qu'il faudrait choisir pour aborder sérieusement le problème.

Le futur parc devrait être établi avant tout pour l'agrément rlu public, et aussi pour la sauvegarde de la végétation spontanée qui y subsiste. Une surveillance suffisante devrait pouvoir éviter le retour d'incendies comme celui dont les traces sont encore visibles. Et rien n'empêcherait de développer les reboisements entrepris sur le rebord des Causses.

Les touristes pourraient séjourner dans de charmants hôtels ou chalets, tel le Château de la Caze, ravissant manoir fortifié du $\mathrm{xv}^{\mathrm{e}}$ 
siècle (des emplacements seraient réservés à ceux qui préfèrent camper). Ils devraient avoir la faculté de naviguer sur le Tarn et de s'y livrer à la pêche à la ligne, quitte à ce que soit assuré un certain repeuplement. Or, à l'heure actuelle, le cours d'eau appartient aux riverains qui peuvent interdire de telles pratiques, susceptibles cependant d'attirer des visiteurs.

La chasse par contre ne serait pas tolérée; et l'on pourrait caresser l'espoir de voir réapparaître la légendaire " bête du Gévaudan ) qui ne serait guère dommageable qu'aux brebis caussenardes.

Peut-être un droit d'entrée modeste permettrait-il de couvrir les frais de gardiennage et une partie de ceux d'aménagement, ces derniers d'ailleurs réduits au minimum.

Que les camarades méridionaux veuillent bien réfléchir à cette question s'ils le jugent à propos.

L. Duplaquet.

\section{Une richesse inexploitée: les hêtres du Sundgau}

Le Sundgau se trcuve situé dans le département du Haut-Rhin, entre Mulhouse et la frontière suisse. C'est un pays de petites collines - les derniers contreforts du, Jura - Les prairies occupent les fonds, de belles futaies de Hêtre s'étendent sur les vallonnements. Le sol calcaire avec des stations loessiques, favorables à la végétation forestière, donne une production vigoureuse (annuellement plus de $6 \mathrm{~m}^{3}$ à l'hectare), les hêtes sont de qualité moyenne et supérieure à celle des Vosges.

L'exploitation de ces forêts est cependant assez délaissée, bien que les rendements dans les coupes existantes soient élevés: de 6 à $7 \mathrm{~m}^{3}$ par hectare (moyenne française: 3 à 4 , moyenne suisse: 4 à 5 ). Il n'existe aucune usine importante dans la région pour utiliser une telle matière première. Les $80 \%$ des coupes sont transformés en bois de chauffage.

\section{Pourquor?}

En dehors des causes plus ou moins directement liées à la guerre et aux situations qui tiennent de l'histoire, il faut savoir que les communes sont pour les deux tiers propriétaires de ces forêts. Elles n'ont pas toujours vu l'intérêt que présentait l'exploitation de ce capital. Dans ce coin deFrance au particularisme accentué, on tient à maintenir intégralement son capital par souci de thésaurisation. Le bois de feu se vend très cher. A quoi bon alors faire des grumes? Mais la diminution de la consommation du bois de feu est amorcée. Les communes vont de ce fait chercher d'auties débouchés si elles tiennent à maintenir au même niveau leurs ressources.

\section{DES RERSPECTIVES NOUVELLES}

Depuis peu, sous l'impulsion du Service des Eeaux et Forêts et de diverses personnalités, les conditions économiques du département dégagent de nouvelles perspectives:

Les communes s'orientent vers l'exploitation plus rationnelle, elles vont se grouper en syndicats intercommunaux pour l'achat en commun de matériel 